A N N A L E S Annales de Bretagne et des Pays de l'Ouest

\title{
La recherche à l'université de Tours de 2001 à 2007
}

Liste des thèses et mémoires soutenus et déposés à l'université de Tours

\section{(2) OpenEdition}

\section{Journals}

Édition électronique

URL : http://journals.openedition.org/abpo/255

DOI : $10.4000 / a b p o .255$

ISBN : 978-2-7535-1512-3

ISSN : 2108-6443

\section{Éditeur}

Presses universitaires de Rennes

\section{Édition imprimée}

Date de publication : 31 décembre 2008

Pagination : 370-377

ISBN : 978-2-7535-0808-8

ISSN : 0399-0826

\section{Référence électronique}

"La recherche à l'université de Tours de 2001 à 2007 », Annales de Bretagne et des Pays de l'Ouest [En ligne], 115-4 | 2008, mis en ligne le 31 décembre 2009, consulté le 14 septembre 2020. URL : http:// journals.openedition.org/abpo/255

Ce document a été généré automatiquement le 14 septembre 2020

(c) Presses universitaires de Rennes 


\section{La recherche à l'université de Tours de 2001 à 2007}

Liste des thèses et mémoires soutenus et déposés à l'université de Tours

1 Précédente publication : ABPO 108-2 (2001), période 1996-2000.

\section{BIBLIOGRAPHIE}

\section{Maîtrises}

2001

ANCHIER, Mickaël, Les relations « internationales » de la Pologne de 966 à la crise des années 1030, dir.

P. DEPREUX.

BARIAUD, Christine, Les maîtres épiciers-ciriers-chandeliers de Tours au XVIIIe siècle. Dynamisme d'une communauté, dir. N. COQUERY.

BIGOT, (Florence), Les mariages à Tours au XVIIe siècle, dir. D. TURREL.

BLOND, Stéphane, Recherches et considérations sur la population de Loches au XVIIIe siècle, dir.

B. MAILLARD.

BORDAT, Virgile, La municipalité de Châteauroux sous l'Occupation 1939-1942, dir. M. CoINTET.

BOUGRAT, Jérémy, Charles de Gaulle et la grâce des condamnés à mort de l'Épuration, 1944-1946, dir.

M. COINTET. 
BOURDIN, Marylin, Le mariage à Chartres au XVIIIe siècle (législation matrimoniale, éléments et description de la cérémonie, dir. D. TURREL.

CAILLÉ, Thomas, Les païens dans la correspondance d'Augustin, dir. Ch. HUGONIOT.

CHAUvin, David, Aurélien (270-275), dir. B. BEAUJARD.

CHEVALIER, Gérald, Histoire des Écoles de la Chambre Syndicale de la Couture Parisienne, dir. R. BECK et L. KAMITSIS.

COLLARD, Christophe, Les commerces de la rue Nationale à Tours entre 1880 et 1914, Dir. J.-F. ECK.

DEPIGNY, Gérald, La Résistance dans le Cher (1940-1944), dir. M. CoINTET.

DESMAREST, Hélène, L'activité théâtrale dramatique face aux politiques publiques de la culture dans la ville de Tours, 1977-2000, dir. M. COINTET.

DRIDI, Amel, Les femmes face à la justice en Indre-et-Loire de 1876 à 1890, dir. S. APRILE.

DROUAULT, Célia, Le droit et les devoirs des femmes à la période moderne selon la coutume de Touraine, dir. B. MAILLARD.

DufoIs, David, L'action de la municipalité de Jean Royer à l'égard de la jeune dans le cadre des foyers de quartier, 1959-1995, dir. M. CoINTET.

Dumont, Benoît, Gestion municipale et affaires royales au XVIe siècle : les délibérations de Tours de 1517 à 1527, dir. D. TURREL.

DURIS, Philippe, Prison et prisonniers à la fin du XVIIIe siècle. La prison royale de Tours (1780-1786), dir.

B. MAILLARD.

FAUVINET, Michaël, Présentation synoptique d'un manuscrit inédit rédigé par Donatien Marie-Joseph de Vimeur, vicomte de Rochambeau, entre 1787 et 1790, dir. B. MAILLARD.

FonTAINE, Alexandre, Étude archéozoologique de l'habitat protohistorique du Petit Chauvort (commune de Verdun-sur-le-Doubs, Saône-et-Loire), dir. M.-P. HORARD-HERBIN.

FOUCHER, Bénédicte, La figure du prince à travers Le Mercure de France, dir. D. POULOT.

FRIGOT, François, U.N.R. et gaullisme électoral en Indre-et-Loire : 1958-1974, dir. M. CoINTET.

GARDIN, Mélanie, Iulia Mamaea, impératrice syrienne, mère de l'empereur romain Sévère Alexandre, dir. M. SARTRE.

GERMINET, David, Étude de la faune d'un habitat rural du Second Age du Fer en Vendée : le site du Grand Paisilier, dir. M.-P. HORARD-HERBIN.

GoBIN, Aurélie, Analyse topographique et évaluation du potentiel archéologique du secteur Anatole France/Saint Saturnin à Tours, dir. H. GALINIE.

Goumy, Steve, Étude géomorphologique d'un vallon en milieu cristallin dans le Massif Central français par une approche archéologique d'Anderitum à Javols, dir. A. FERDIERE.

HABERT, Vincent, Meuniers et moulins à eau de la Sologne romorantinaise dans la première moitié du XIXe siècle, dir. S. APRILE.

HAMMON, Olivier, L'émergence des mouvements sportifs à Chartres au tournant du XIXe siècle (1877-1914), dir. J.-F. ECK.

HESSARD, Jean, Connaissance et représentation du monde selon Ibn Khaldûn, dir. C. BOUSQUET-LABOUÉRIE. 
JEAN, Natascha, Les transformations du paysage à trois échelles de temps dans le secteur de BransDammartin (Jura), dir. G. CHOUQUER.

JEANDROT, Aude, Étude de la pêcherie du prieuré du Foncher au Moyen Âge (Xe-XVe siècle), dir. J. BuRnouf et E. ZADORA-RIO.

JeAnnin, Cécile, Le couvent des Viantaises à Beulieu-Lès-Loche, 1643-1793, dir. G. CHAIX.

JURADO, Marlène, La fédération d'Indre-et-Loire du Rassemblement pour la République, dir. M. COINTET.

LEMAS, Nicolas, Le projet urbain des Lumières et sa destinée. Delamair, Dussaussoy, Laugier, Patte, Poncet de la Grave, ou l'invention d'un urbanisme, 1730-1770, dir. D. РолLoT.

LoustALot, Arnaud, La guerre d'indépendance turque vue par Le Temps, L'Humanité, et LaCroix, d'un armistice à l'autre (1918-1922), dir. J.-M. MoINE.

Melado, Tony, La vie de saint Maur. Extrait du manuscrit 20 de la Bibliothèque municipale de Tours, Vita Sancti Mauri et Miracula Sancti Mauri par l'abbé Odon de Glanfeuil : transcription et commentaire, dir. B. JUDIC.

MıcноU, Stéphanie, Fidélité personnelle et loyalisme monarchique : les Souvré (fin XVIe-début XVIIe siècle), dir. K. BEGUIN.

PAULAY, Cédric, Concours grecs et coutumes agonistiques en Syrie, dir. M. SARTRE.

Peu-Duvallon, Jonathan, La paroisse de Saint-Denis d'Amboise et la dysenterie de 1779, dir. G. ChaIX.

PILLAULT, Sophie, Étude architecturale et fonctionnelle d'un bâtiment gallo-romain : la structure 1 de J.S.F (Javols/Lozère), dir. A. FERDIERE.

POUTEAU, Julien, La hiérarchie des fortunes en Indre-et-Loire en 1848, dir. J.-F. EcK.

REDOUIN, Damien, Une presse des Lumières et une presse d'information locale à Tours en 1785 et 1786 : les Affiches de Touraine, dir. N. COQUERY.

REGNIER, Nelly, DionChrysostome et la politique des cités, dir. M. SARTRE.

RULLON, François, L'exode en Indre-et-Loire. Septembre 1939-septembre 1940, dir. M. COINTET.

SAlIN, Marilyne, Étude de la faune du fossé défensif du Bas-Empire d'un site de Tours (site 6), dir. M.-P. HORARD-HERBIN.

SFIA, Nadia, Une seigneurie dans la seconde moitié du XVIIIe siècle : la baronnie de Veretz, dir. K. BEGUIN. THAON, Aurélie, Philippe l'Arabe (204-249 apr. J.-C.), dir. M. SARTRE

THомаs, Guillaume, La Commune de Paris vue par un quotidien bonapartiste : Le journal de L'Indre-etLoire, 1871-1880, dir. J.-M. MoINE.

TOSNA, David, L'image de l'évêque à travers l'épigraphie wisigothique du vIe au vIIIe siècle, dir.

N. GAUTHIER

VASSELIN, Fabien, Démographie des entreprises à Tours et en Indre-et-Loire, 1843 1900, dir. M. LESCURE. VEYRET, Julien, Les instituteurs publics d'Indre-et-Loire dans le mouvement syndical de l'entre-deux guerres, dir. R. BECK.

VILLA, Audrey, Hermione Quinet, une correspondance féminine d'exil, 1852-1870, dir. S. APRILE.

VILLETTE, Laurie, La Libre Pensée en Indre-et-Loire sous les Quatrième et Cinquième Républiques, dir. M. COINTET. 
WATISSEE, Matthieu, La Huitième Légion Auguste, dir. B. BEAUJARD.

\section{2}

ABADIE, Julien, L'élection présidentielle américaine de novembre 2000 vue par les médias français, dir.

P. CHASSAIGNE.

ANCELET, Isabelle, L'hippologie au XIIIe et XIVe siècle selon Jordanus Rufus et le Nâsiri : entre Occident et Orient, dir. P. DEPREUX.

ANDURAND, Olivier, Deux archevêques de Tours face au jansénisme : François Blouet de Camilly (1720-23), Louis Jacques de Chapt de Rastignac (1723-50), dir. G. CHAIX.

AVRIL, Flavie, L'île de Samothrace et le culte des Cabires dans l'Antiquité grecque, dir. P. BRUN.

BARANGER, Christophine, Étude iconographique des vitraux du déambulatoire supérieur de chœur de la cathédrale du Mans, dir. C. BOUSQUET.

BEGUIN, Magali, Typo-chronologie des sépultures et pratiques funéraires sur le site de Rigny-Ussé (Indreet-Loire) : la partie nord du site (zones 1,2,3,7 et 8), dir. E. ZADORA-RIO.

BESSON, Aline, L'île d'Amorgos dans l'Antiquité, dir. P. BRUN.

BLANCHET, Céline, La prison de Loches sous le Second Empire, dir. S. APRILE.

BOSSEAU, Romanella, Analyse topographique et évaluation du potentiel archéologique de trois îlots du centre urbanisé ancien à Tours (secteur de la cité), dir. H. GALINIÉ.

BoUTAUD, Benoît, Le proche entourage de François Mitterrand.1916-1981, dir. M. CoINTET.

CAILlEAU, Willy, Une marine de pierre : étude des graffiti naviformes de la vallée de la Vienne et de la Creuse, dir. B. MAILLARD.

CHASSEL, Marie-Laure, Écoles et éducation en Gaule romaine, dir. B. BEAUJARD.

CHAUVET, Julie, Lespratiques religieuses dans les tragédies conservées de Sophocle, dir. S. MILANEZI.

COLLET, Vincent, Espace social et pratiques culturelles de la noblesse blésoise dans la seconde moitié du Siècle des Lumières, dir. G. CHAIX.

DEMAREST, Mélanie, Étude du mobilier métallique du site gaulois et gallo-romain de Touffréville « La Saussaye «(Calvados), dir. A. FERDIÈrE.

DENIE, Carole, La forêt d'Amboise au XVIIIe siècle, dir. B. MAILLARD.

DHENIN, Guillaume, La vie religieuse dans les campagnes en Gaule au IVe siècle, dir. B. BEAUJARD.

Dumont, Jean-Marie, Le « Sillon « de Marc Sangnier : une minorité d'élite, dir. M. COINTET.

DURAND, Benjamin, Musique et cultures rock en Angleterre de 1966 à 1974, dir. R. BECK.

DuvAllet, Mickaël, Les activités portuaires du Pirée durant l'époque classique, dir. J.-C. CoUveHNES.

FILlon, Aurélie, Étude d'un incunable de 1499, Tractado de la vida y estado de la perfeccion, dir. M.C. GERBET.

FOUASSIER, Stéphane, L'histoire de la Gaule revisitée par la bande dessinée d'Astérix le Gaulois, dir. B. BEAUJARD et M. COINTET.

GILOT (Alexia), La justice seigneuriale de la châtellenie de Cormery, 1745-1749, dir. B. MAILLARD. 
GOUSSARD, Céline, Laville de Tours à travers ses comptes municipaux (1527-1544), dir. D. TURREL.

HAMDI, Hayatt, La gestion municipale communiste de Saint-Pierre-des-Corps (1919-36), dir. J.L. PINOL.

HANANY, Angélique, La criminalité féminine en Indre-et-Loire de 1900 à 1939 : les crimes de sang, dir.

P. CHASSAIGNE.

HUARD, Matthias, L'empereur Commode (161-192), dir. B. BEAUJARD.

JEULIN, Mickaël, Le berger grec, personnage des frontières (époques archaïque et classique), dir.

S. MILANEZI.

LAMARCHE, Aurélie, Nero et Drusus Caesares : la vie des deux fils aînés de Germanicus, de leur naissance à leur réhabilitation posthume (5-41 apr. J.-C.), dir. F. HURLET.

LAURENT, Amélie, Application du pénétromètre dynamique de type PANDA en archéologie urbaine:

l'exemple du site de Saint-Julien à Tours, dir. H. GALINIÉ.

LEMESLE, Hélène, Vautours, singes et cloportes. Propriétaires, entrepreneurs locataires et gérants des villes et des champs dans les archives privées de Ledru-Rollin (1807-1874), dir. S. APRILE.

MARIC, Claire, La participation politique des femmes en Angleterre au début des années 1980 (élections générales de 1983), dir. F. CHAUBET.

MEES, Virginie, Marie-Antoinette et l'Affaire du collier, à travers les écrits de la fin du XVIIIe jusqu'au XXIe siècle, dir. B. MAILLARD.

MEMETEAU, Nicolas, La guerre de siège dans l'œuvre de Thucydide, dir. P. BRUN.

MEUNIER, Stéphanie, Délos à l'époque classique, dir. P. BRUN.

NiCOLAS, Virginie, Biographie du chanoine Noël Carlotti (1900-1966), dir. M. COINTET.

NOBLET, Jean-Philippe, Une petite paroisse du pays chartrain au XVIIIe siècle, Ermenonville-la-Grande. Société, culture et population, dir. B. MAILLARD.

PEREZ LOPEZ, Rémi, Les communautés allophones de la ville de Québec à la fin du XIXe siècle, Étude des stratégies d'intégration, dir. S. APRILE.

POIRIER, Nicolas, L'implantation des sites ruraux dans le Sancerrois antique : facteurs naturels et facteurs humains, dir. A. FERDIÈRE.

POLASZEK, Christophe, Le « pain quotidien « à Tours dans la seconde moitié du XVIIIe siècle. Surveillance et conflits, dir. B. MAILLARD.

POUFfarin, Céline, La politique d'hygiène publique à Tours (1849-1902), dir. J.-L. PINOL.

QUEVILLON, Sophie, Analyse spatiale de la ville antique de Vieux (Calvados) : le S.I.G. comme outil d'analyse de l'espace urbain, dir. A. FERDIERE.

REROLLE, Charlotte, Iconographie des dieux à Pétra et à Palmyre : étude comparée.(Ier siècle av. J.-C.-IIIe siècle apr. J.-C.), dir. M. SARTRE.

RoUHAULT, Linda, Étude architecturale de la structure 2 (l'éventuel logis du XIVe-Xve siècle) du château de Rochefort-en-Terre (Morbihan), dir. A. NISSEN JAUBERT.

RouSSELET, Aurélien, Prédication et petites écoles dans le diocèse de Tours sousl'Ancien Régime, dir.

G. CHAIX.

SERIER, Laurence, Une ville et son fleuve : l'exemple de Tours au XVIIIe siècle, dir. B. MAILLARD. 
SINETY, Renaud de, La vision des « indigènes » subsahariens dans la seconde moitié du Xve siècle d'après trois récits de voyage, dir. S. BOISSELIER.

TROUBADY, Murielle, Apport du matériel monétaire à l'étude des sites gaulois de la forêt de Compiègne, dir. M.-P. HORARD-HERBIN.

TRUILLET, Jonathan, Les appelés du contingent et le souvenir de la guerre d'Algérie. Une mémoire apaisée ou conflictuelle?, dir. M. COINTET.

\section{3}

ARRIVE, Sophie, Regards sur la construction du passé dans les contes et nouvelles du XVIe siècle, dir.

F. ALAZARD.

BARATIN, Raphaël, La diffusion de la micro-informatique personnelle en France de 1973 à 1995, dir. J.-

L. PINOL.

BIERE, Yves, Étude des éléments ferreux des ateliers dits du « charpentier « et « de l'huilerie « à Jerash (Jordanie), dir. J. SEIGNE.

BOUET, Cécile, Étude d'une partie de la faune du site protohistorique du C.H.U. Clocheville de Tours (Tène C2-D1), dir. M.P. HORARD-HERBIN.

BOUSSES, Vincent, Gendarmerie et populations civiles de l'Indre entre 1939 et 1944 (à partir des archives de la Gendarmerie Nationale), dir. M. CoINTET.

BRISEUX, Laure, Les Hortensii, histoire d'une famille, dir. B. BEAUJARD.

BRUNEL, Raphaël, Le cinéma social en France depuis les années soixante-dix, dir. P. CHASSAIGNE.

CHARBONNIER, Laëtitia, La sidérurgie en Bas-Berry : les forges de Clavières au XVIIIe siècle, dir.

B. MAILLARD.

CHERY, Cécile, L'alimentation en eau des villes de Gaule à l'époque romaine, dir. B. BEAUJARD.

CHOU, Frédéric, La ville et le sang. Les Abattoirs de Tours au XIXe siècle, dir. R. BECK.

CUVIER, Raphaël, La notabilité tourangelle sous le Second Empire, dir. J.-L. PINOL.

DALLE, Yann, Impérialisme et cinéma des États-Unis. Les films américains en France de 1945 à 1995, dir.

R. BECK.

DeloM, Nicolas, Étude de quelques ensembles céramiques protohistoriques du site de Clocheville (Tours, Indre-et-Loire, 37), dir. M.-P. HORARD-HERBIN.

Ducongé, Sébastien, Les poteries de 2e Âge du Fer de la grotte des Perrats à Agris (Charente). Apport à l'interprétation des occupations du site au cours de la Tène, dir. M.-P. HORARD-HERBIN.

FAROUELLE, Sébastien, Crimes et criminalité à l'écran, 1920-1939. France, États-Unis, Grande-Bretagne, Allemagne, dir. P. CHASSAIGNE.

GASTON, Sébastien, L'insécurité dans le Lochois durant l'été 1944 : l'affaire du maquis Le Coz, dir.

M. COINTET.

GOUACHE, Renaud, La France et les Jeux Olympiques de 1936, dir. R. BECK.

GOUBARD, Emmanuelle, Histoire de la paroisse Notre-Dame-la-Riche, à Tours, de la signature du Concordat jusqu'aux débuts de la Monarchie de Juillet, dir. J.-M. MoINE. 
KerRos, Nathalie, Une famille de la noblesse blésoise : les Belot, dir. B. MAILLARD et M. VERGÉ-FrANCESCHI. LARUAZ, Jean-Marie, Les oppida du département de l'Indre-et-Loire, dir. A. FERDIERE.

LEBORGNE, Nicolas, L'art de naviguer en France au XVIe siècle, dir. P. BRIOIST.

LEPROUST, Guillaume, Le crime d'argent en Indre-et-Loire (sondages 1825-1830, 1845-1850, 1865-1870, 1885-1890), dir. P. CHASSAIGNE.

LEROUX, Fabrice, La géographie d'Ousama Ibn Mounkidh, dir. C. BOUSQUET.

MERRIEN, Caroline, La fabrication pamphlétaire d'un acteur de la Fronde : la harangère des Halles, dir. K. BEGUIN.

NEUVY, Laëtitia, La municipalité de Tours, la mère et l'enfant. Organisation de la protection maternelle et infantile de 1930 à 1970, dir. J.-M. MoINE.

PERIS, Julia, La condition féminine à Tours de 1967 à 1977, dir. R. BECK.

Pigeolet, Sébastien, Azé, une paroisse du Perche Vendômois. La vie quotidienne de ses habitants dans la deuxième moitié du XVIIIe siècle (1753-1788), dir. B. MAILLARD.

REYTIER, Richard, Un siècle de pêche de loisir en Touraine, dernier tiers du XIXe siècle-dernier tiers du XXe siècle, dir. R. BECK.

Roncin, Sébastien, Ernest Mame (1805-1883). L'ascension politique d'un imprimeur de Tours de la mairie au corps législatif, dir. J.-M. MoINE.

SARRESTE, Florian, La sidérurgie ancienne dans la nord-ouest du Berry, dir. A. FERDIERE.

SERVANT, Angélique, Les instituteurs en Indre-et-Loire de 1815 à 1880, dir. R. BECK.

SPITALERI, Sandrine, Reconstruire la basilique Saint-Martin : projets et querelle, 1861 1895, dir.

M. COINTET.

SURGENT, Amandine, Les édiles et les jeux publics, de la fin de la première guerre punique à la fin de la République, dir. C. Hugoniot.

TARGET, Anne, Les duels en France de 1877 à 1906 : pratiques, rouages et mécanismes, dir. P. CHASSEIGNE.

VALLEE, Matthieu, Les soieries tourangelles et leur marché, 1850-1914, dir. J.-M. MoINE.

VARACHEIO, Sylvia, Sacré et profane dans les fêtes florentines au XVIe siècle, dir. F. ALAZARD.

VIARD, Rudy, Les guerres de Six Jours et du Kippour. Représentations comparées de deux événements militaires dans la presse française (Le Monde, Le Canard Enchaîné et Minute), dir. M. LARGEAUD.

WOODLAND, Sandrine, «Tours, ville royale « au temps de la Ligue (1589-1595), dir. D. TURREL.

\section{Master 1}

\section{4}

ACCARIES, Elodie, Le cheval au Ive siècle avant Jésus-Christ : La vision de Xénophon, dir. COUVENNES. ADAM, Barbara, Des sages-femmes, des femmes sages? Etudes de la création d'une profession et de la naissance d'un groupe social : les sages-femmes tourangelles au XVIIIe siècle, dir. D. TURREL

ANDRAULT, Véronique, La naissance et l'évolution de la vis dans les pressoirs à vin et à huile en Syrie antique, dir. J. SEIGNE. 
ARRETEAU, Irène, Recherches sur l'iconographie de saint Martin à travers les vitraux martiniens (XIIIe-XIVe siècles) des cathédrales gothiques de Tours et de Chartres, ainsi que quelques enluminures tourangelles, dir. C. Bousquet.

BERANGER, Katia, Les paysans dans la littérature toscane des XIVe et XVe siècles, dir. N. BOULOUX.

BERDAL, Virginie, Les Pythia, leurs épreuves musicales et leurs pythioniques (des origines à l'époque hellénistique), dir. S. MiLANEZI.

BIGoT, Stéphanie, Étude du mobilier métallique protohistorique (la Tène C2-D1) du CHU de Clocheville à Tours (37, Indre-et-Loire), dir. M.-P. HORARD-HERBIN.

BOUSQUET, Stéphanie, Les lieux d'échange dans le sud de la Scandinavie durant le premier millénaire et plus particulièrement les VIIIe-XIe siècles, dir. A. NISSEN JAUBERT.

BRAS, Virginie, La carrière de professeur aux IVe et ve siècle après J.-C., dir. B. BEAUJARD.

CHALON, Dorothée, Les pratiques religieuses des parisiens au début du Xve siècle. dir. N. BoulouX

CORMIER, Frédérique, Ninon de Lenclos dans le « Siècle des dévots » (1623-1705), dir. M. VERGÉ-FRANCESCHI. COUDREAU, Yann, Les violences interpersonnellles à Tours, 1919-1939, dir. P. CHASSAIGNE.

COURTOIS, Julien, Gués et ponts antiques dans le territoire de la cité des Turons, dir. J. SEIGNE.

DABEK, Pierre, Les structures de stockage de la région Centre au haut Moyen-Age, dir. A. NISSEN-JAUBERT

DALloIs, Mélanie, Les Merveilles dans l'œuvre de Gervais de Tilbury, lesOtia Imperialia, Tertia Decisio, dir. N. Bouloux.

DRIARD, Cyril, Recherches sur l'alimentation en eau de Caesarodunum. L'aqueduc antique du Cher, dir. J. SEIGNE.

DUBOIS, Romain, Batellerie et commerce de Loire. Voituriers par eau et marchands fréquentant la rivière de Loire, XVIe-XVIIe siècles, dir. M. VERGÉ-FRANCESCHI.

GaUvin, Caroline, Lieu de loisir, lieu de pédagogie : le jardin botanique de Tours. Histoire d'une institution, dir. R. BECK.

GoDIGNON, Denis, Pratiques funéraires du Haut-Empire à Javols-Anderitum d'aprèsl'étude de cinq sépultures, dir. A. FERDIÈRE.

GUILLEMARD, Thomas, La céramique de l'atelier de potier gallo-romain de Vrigny(Loiret), dir. A. FERDIÈRE. HELLY-LE BOT, Perrine, Rome et la reconstruction des cités en Asie Mineure d'Alexandre à Sévère Alexandre, dir. M SARTRE, B. POUDERON.

HERVET, Hugues, La navigation à Tours au XIXe siècle, dir. R. BECK.

JAFFROT, Étienne, La céramique : élément de caractérisation du sol urbain. Étude de cas : le site de SaintJulien à Tours Indre-et-Loire, dir. H. GALINIÉ.

JOUVEAU, Aurélia, Le Tour de France cycliste en Indre-et-Loire, 1955-2000, dir. R. BECK.

LE COUEDIC, Mélanie, La montagne d'Enveig (66) : mise en œuvre des données archéologiques, environnementales et planimétriques à l'aide d'un SIG, dir. E. ZADORA-RIO.

LEREBOURG, Laetitia, L'enfant au travers de l'émission de radio de Menie Grégoire en 1979, dir. M. COINTET. LUQUET, Guillaume, Les archevêques de Bourges et la réception des conciles œcuméniques au XVIe siècle, dir. G. CHAIX et. P. BRIOIST. 
MALJEAN, Denis, Archéologie minière, méthodes et limites : le cas des mines « du Bleymard », dir. B AILLYMAÎTRE, dir. A. FERDIÈRE.

MALJEAN, Denis, Canons et fondeurs en Touraine aux XVe-XVIe siècles. Étude locale sommaire et pièces remarquables de la ville de Romorantin (Cher), dir. P. BRIOIST.

MASSON, Aurélie, La chronologie de la Grande colonnade de Palmyre : la section centrale à partir des faits et publications existants, dir. J. SEIGNE.

MASSON, Edouard, L'exploitation du castor par les Iroquoiens de la vallée du Saint Laurent et du Sud-Ouest de l'Ontario entre 1350 et 1650 apr. J.-C. dir. M.-P. HORARD-HERBIN.

MEUNIER, Elodie, Euvre de charité ou institutions hospitalières? Assistance et population secourue dans le Berry aux XVIe et XVIIe siècles : trois hôtels-Dieu représentatifs (Vierzon, Issoudun et Bourges), dir.

D. TURREL.

NiCLOT, Marion, Reconstitution du paléoenvironnement et du paléoclimat d'après les rongeurs du site mousténien des Pradelles, à Marillac-le-Franc (Charente), dir. M.-P. HORARD-HERBIN.

NOLEAU, Nicolas, Inventaire informatisé et raisonné des vestiges archéologiques de Luynes antique, dir. J. SEIGNE.

PAPIN, Pierre, Étude de la topographie historique du Néolithique à la période su contemporaine à l'échelle d'une commune rurale : Le Grand-Pressigny (Indre-et Loire), dir. E. ZADORA-RIO.

PesCHer, Benoît, Le petit mobilier de la Tène dans le Berry, dir. M.-P. HORARD-HeRBIN.

PoIRSON, Renaud, Les nomades et la loi du 16 juillet 1912 en Indre-et-Loire (1913 1923). La mise en ceuvre de la loi. La création d'une population, dir. S. APRILE.

RENAULT, Nicolas, Le glam rock et la jeunesse britannique (1971-1975), dir. P. CHASSEIGNE.

SOPPELSA, Caroline, Le Pèlerinage de Vie humaine de Guillaume de Digulleville, du texte à l'image. Étude iconographique réalisée à partir de trois manuscrits et d'un incunable de la fin du Moyen Âge, dir. C. BOUSQUET-LABOUERIE.

VISTEL, S., Les voies romaines dans le nord de la cité des Turons. État actuel de la documentation et réalité physique de la voirie antique, dir. J. SEIGNE.

VoLANT, Julie, Les scies à encoches de la région pressignienne comparée à celles découvertes dans d'autres régions : analyse typologique et technologique, dir. M.-P. HORARD-HERBIN.

\section{5}

AIRoLA, Céline, Les femmes et l'argent à l'époque hellénistique, dir. C. GRANDJEAN.

BEURTHERET, Maude, Les lieux d'inhumation en Indre-et-Loire du vie au xe siècle : approche spatiale, dir. E. LORANS.

DA CostA, Nicolaï, Conjoncture historique et profession judiciaire : un avocat à Tours sous l'occupation, dir. M. COINTET.

DEGORRE, Emeline, La sculpture figurée en Berry antique (départements du Cher et de l'Indre), dir.

A. FERDIÈRE.

FAUSSURIER, Bérengère, Quelques aspects épistolaires et littéraires de l'épisode tourangeau de la vie de Jacques-Auguste de Thou : politique, religion et culture, dir. F. ALAZARD. 
MALEPERT, Sébastien, Les années Lang à Blois, dir. M. COINTET.

MAROT, Emeline, Évolution topographique du monastère de Marmoutier (Tours, Indre-et Loire) du IVe au XIXe siècle. Élaboration d'un Système d'Information Géographique, dir. E. LoRANS.

PERCHENET, Virginie, Le système d'information italien dans la Gazette de Théophraste Renaudot 1631-1648, dir. F. ALAZARD.

Peyne, Nicolas, La céramique de Saint-Gence (Haute-Vienne) - Campagnes de fouille de 1998 et 1999. Au lieu-dit « Le Pâtureau », dir. A. FERDIÈRE.

PIRAUDEAU, Benoît, La Gestapo et ses collaborateurs français en Touraine (1942-1944), dir. J.F. ECK.

QUILEZ, Emeline, Étude de la faune de la Tène ancienne du site de "La Chaussée de Chappe ». (Bourges, Cher), dir. M.-P. HORARD-HERBIN.

SOREL, Yoann, La verrerie du site gallo-romain de Montaigu-la-Brisette (Manche), agglomération secondaire de la cité des Unelles, dir. A. FERDIÈRE.

SURGENT, Marianne, L'alimentation en eau de Bourges dans l'Antiquité : état des connaissances sur l'aqueduc de Traslay, dir. J. SEIGNE.

TURBAN, Karine, La contrebande de tabac et d'allumettes dans les centres urbains du Loiret et d'Indre-etLoire au XIXe siècle (1810-1906), dir. S. APRILE.

\section{6}

AGEN, Grégory, Le massacre d'Oradour-sur-Glane et le procès de Bordeaux, dir. M. COINTET.

BANCAUD, Aude, et BODY, Mathilde, Étude architecturale du « régulier » du prieuré Saint-Cosme (La Riche, Indre-et-Loire), dir. E. LORANS.

BARDOU, Émilie, Marie Du Bois, un valet de chambre de Louis XIV à la cour, d'après ses Mémoires (1647-1676), Dir. B. PIERRE.

COUTURIER, Yann, Le quartier Saint-Maurice de Chinon : mise en place d'une base de données documentaire et d'un Système d'information Géographique, dir. E. LORANS. DAVID, Antoine, Les réfugiés polonais en Indre-et-Loire, 1831-1864, dir. S. APRILE.

Duvoux, Stéphanie, Les guerres de religion cinquante ans après : regards des historiens français de la première moitié du XVIIe siècle sur un épisode récent, dir. F. ALAZARD.

LUZET, Coraline, Le bourg d'Angers du xxe au xve siècle. Topographie historique et mise en place d'un Système d'Information Géographique, dir. E. LoRANS.

MARTINEAU, Fabien, Les acteurs de l'école primaire en Indre-et-Loire pendant l'entre deux guerres (1919-1939), dir. S. APRILE.

QUERU, Marie, Les procès-verbaux d'estimation des biens nationaux du quartier canonial de Tours: élaboration d'une base de données et analyse de l'évaluation, dir. E. LoRANS.

Roux, Émilie, L'organisation spatiale du Puy-de-Corent : un aperçu de 150 ans de recherches archéologiques, dir. S. FichTL et M. Poux.

SAINT-DIDIER, Guillaume, L'artisanat des métaux à Javols-Anderitum (Lozère) à l'époque romaine : structures et déchets de production, dir. S. FICHTL.

SEBILLE, Anne-Pauline, Portillon, un quartier industriel tourangeau (1830-1940), dir. J.-M. MoINE. 
TESSIER, Alexandre, Le phylloxéra en Touraine, dir. M. DE FERRIERE LE VAYER.

VILLERET, Maud, Le commerce alimentaire à Tours au XVIIIe siècle, dir. N. COQUERY.

\section{7}

HENRI, Delphine, Apports à la connaissance des sépultures découvertes dans l'église abbatiale de Marmoutier (Tours, Indre-et-Loire) : étude préliminaire des toiles etsergés mis à jour lors des fouilles anciennes, dir. E. LORANS.

FEUILLASSIER, Benoît, Les monnaies mérovingiennes frappées dans le diocèse de Tours (558-750). Catalogue et analyse préliminaire, dir. E. LORANS.

DESCHAMPS, Marion, VANTOMME, Marion, Architecture civile médiévale à Tours : analyse archéologique d'une propriété de Châteauneuf (12-14 rue Paul-Louis Courier, dir. E. LoRANS.

BouYssou, Gerbert-Silvestre, Commentaire historique du Hiéron de Xénophon, dir. J.-C. CouvenHES.

LLIBOUtRY, Matthieu, Rôle et action des Capucins dans la Contre-Réforme catholique à Prague et en Bohême durant le XVIIe siècle, dir. L. KLUSAKOVA et B. PIERRE.

BRIANT, Romain, Chinon, Histoire de la première centrale nucléaire française, de sa création à nos jours, dir. M. DE FERRIÈRELE VAYER.

\section{Master 2}

\section{5}

BOUSQUET, Stéphanie, Les critères urbains retenus par les archéologues protohistoriens et hautmédiévistes en Europe du nord-ouest, dir. GALINIÉ.

CLARPIN Moussounda, Féréole, Analyse typologique et technologique des fragments de lames de silex trouvées sur le site du Petit Paulmy (Indre-et-Loire), dir. FERDIÈRE.

COQUERY, Jean-François, Formes fluviales et formations superficielles dans la vallée de la Vienne de Chinon à Marcilly-sur-Vienne durant l'Holocène, dir. E. ZADORA-RIO.

CoutoIs, Julien, Fondations sur pieux de bois dans l'antiquité : études de quelques cas particuliers situés sur le territoire français, dir. SEIGNE.

LECREUX, Michelle, Le Matinsous l'occupation. Une collaboration ordinaire, dir. COINTET.

PAPIN, Pierre, Étude diachronique de l'occupation du sol à l'échelle micro-régionale : topographie historique de la région d'Athée-sur-Cher (37) des origines à la période sub-contemporaine, dir. E. ZADORARIo.

PESCHER, Benoît, Étude du mobilier métallique gaulois du terrain Charbonnier à Levroux (Indre), dir. O. BUCHSENSCHUTZ.

\section{6}

BRUNEAU, Gérald, La surveillance des anarchistes à Tours, 1892-1914, dir. P. CHASSAIGNE.

CHILMA, Romain, Deux sociétés face au crime : Les tueurs en série en Grande-Bretagne et en France 1880-1950, dir. P. CHASSAIGNE. 
CORBINUS, Elodie, Azay-le-Rideau : un seigneur et sa terre à la fin du Moyen Âge, dir. F.-O. ToUATI.

Dumont, Anne, Les scurs de Charité dominicaines de la Présentation de la Sainte Vierge à Tours au XIXe siècle. Les Dominicaines de la Présentation de Tours de 1809 à 1909, entre autorité et utilité sociale, dir. R. BECK.

LE BiHAN, Alexandre, Les médaillés de Sainte-Hélène dans le Loir-et-Cher (1857-1896), dir. J.-

M. LARGEAUD.

LEMONNIER, Vincent, Les Irlandais employés dans la marine française (1688-1791), dir. M. VERGÉFRANCESCHI.

LEPAGE, Anne-Laure, Le temps liturgique en Hesse sous le règne de Philippe le Magnanime (1518-1567), dir. B. HEYBERGER et H. SCHNEIDER.

MAROT, Emeline, Le site du monastère de Marmoutier (Tours, Indre-et-Loire), analyse archéologique des vestiges antérieurs au XIe siècle, dir. E. LORANS.

NAU, Sylvain, La nouvelle Droite française : pour un nationalisme européen. Le G.R.E.C.E. et la revue éléments (1973-1982), dir. R. BECK.

Peyne, Nicolas, La céramique de Saint-Gence (Haute-Vienne). Campagnes de fouilles de 1998 et 1999 au lieudit « Le Pâtureau », dir. S. FICHTL.

QUILEZ, Emeline, Alimentation carnée et utilisation des matières animales au ve siècle av. J.-C. L'exemple archéozoologique de Port-Sec Sud (Bourges, Cher), dir. M.-P. HORARD-HERBIN.

SILL, Edouard, «Ni Franco, ni Staline ! ». Les volontaires français de la Révolution espagnole. Miliciens et militants révolutionnaires en Espagne 1936-1939, dir. J.-M. LARGEAUD.

ZANON, Aldo, La Croix-Rouge à Tours (1937-1947), dir. J.-M. MoINE.

\section{7}

BARRIER, Sylvie, Le bassin monumental, la « Rue des Caves », le Bâtiment « aux Vases Peints » :

Exploitation du mobilier céramique des fouilles des universités de Madrid et de Saragosse dans le secteur de la Pâture du Couvent (Bibracte), dir. S. FichTL.

DUMONTET, Anthony, Étude céramologique sur le site protohistorique de Bourges (Cher), de la Tène B à la Tène $\mathrm{C}$, dir. S. FICHTL.

GARNIER, Mathilde, Chronique et vision du monde selon Adémar de Chabannes et Raoul Glaber, deux historiens du XIe siècle, dir. N. Bouloux.

MARTEAUX, Flore, COUTURIER, Yann, Forteresse de Chinon (37) : Modèles Numériques de Terrain (MNT), de l'acquisition à l'analyse, dir. B. DUFA ̈.

Musso, Joseph, La découverte d'une culture musicale venue d'ailleurs : le reggae en France de 1974 à 1985, dir. R. BECK.

PoupA, Marie-Aimée, Les édifices religieux dédiés à Saint-Martin sur les routes de Saint-Jacques-deCompostelle, dir. C. BOUSQUET.

Roux, Émilie, Les établissements ruraux de la région de Noyers-sur-Serein à travers l'étude de l'instrumentum : caractérisation des activités et essai de hiérarchisation, dir. S. FICHTL. SAINT-DIDIER, Guillaume, Le travail des métaux à Javols-Anderitum (Lozère) à l'époque romaine : structures et déchets de production, dir. S. FICHTL. 
TRÉGOUËT, Grégory, La conception du pouvoir chez les évêques du Val de Loire. Fin XIe siècle-début XIIe siècle, dir. B. JUDIC.

INDEX

Index géographique : Tours 